\title{
The Effects of Melatonin Intake on Biomarkers of Inflammation and Oxidative Stress in Subjects with Breast Cancer: A Randomized, Double-Blind, Placebo-Controlled Trial
}

\author{
Fatemeh Hashemi \\ Kashan University of Medical Sciences \\ Noushin Mousavi \\ Kashan University of Medical Sciences \\ Reza Manouchehri-Ardakani \\ Kashan University of Medical Sciences \\ Mohammad Hossein Pourhanifeh \\ Kashan University of Medical Sciences \\ Amir Hassan Matini ( $\nabla$ amirhassanmatini@gmail.com ) \\ Kashan University of Medical Sciences
}

Research Article

Keywords: Melatonin, inflammation, oxidative stress, breast cancer

Posted Date: February 8th, 2022

DOI: https://doi.org/10.21203/rs.3.rs-1312393/v1

License: () (7) This work is licensed under a Creative Commons Attribution 4.0 International License. Read Full License 


\section{Abstract}

Background: To our knowledge, data on the effects of melatonin supplementation on biomarkers of inflammation and oxidative stress in subjects with breast cancer are scarce. This research was done to assess the effects of melatonin supplementation on biomarkers of inflammation and oxidative stress in subjects with breast cancer.

Methods: This randomized double-blind, placebo-controlled trial was conducted on 40 subjects diagnosed with breast cancer aged $>25$ years old. Subjects were randomly assigned into two groups to take either melatonin $(n=20)$ or placebo $(n=20)$ for 24 weeks. Inflammation and oxidative stress biomarkers were quantified at baseline and after the 24-week intervention.

Results: After the 24-week intervention, compared with the placebo, melatonin supplementation significantly increased plasma total antioxidant capacity (TAC) $(+21.3 \pm 26.2 \mathrm{vs}$. $-6.3 \pm 34.5 \mathrm{mmol} / \mathrm{L}, \mathrm{P}=0.007)$, and decreased plasma malondialdehyde (MDA) concentrations $(-0.2 \pm 0.3 \mathrm{vs} .+0.1 \pm 0.2 \mu \mathrm{mol} / \mathrm{L}, \mathrm{P}=0.002)$. We did not observe any significant effect of melatonin supplementation on other biomarkers of oxidative stress.

Conclusions: Overall, melatonin intake for 24 weeks in patients with breast cancer had beneficial effects on TAC and MDA levels, but did not affect other biomarkers of oxidative stress.

\section{Background}

Breast cancer is the most prevalent malignancy in females across the world; it approximately accounts for twenty five percent of all women cancers and its prevalence is greater in developed countries (1). In addition, this cancer is the second leading cause of malignancy-related death among females worldwide $(1,2)$. Current breast cancer therapies, including radiotherapy, chemotherapy, and surgery may lack efficiency due to a high relapse risk, drug resistance emergence and poor patient response (3). Therefore, exploring better and more effective therapeutics for breast cancer patients is an essential issue.

Melatonin, a natural neurohormone predominantly synthesized by the pineal gland, play an important role in both the synchronization and regulation of the sleep-wake cycle as well as circadian rhythms (4). In addition to its chronobiotic roles, melatonin possesses complex and different immunomodulatory and oncostatic abilities (5-7). Moreover, changed circadian levels of melatonin, especially a decrease in peak endogenous production, have been shown in relation to diverse cancers (8-10), cognitive impairment and healthy ageing $(11,12)$. Notably, the role of inflammation and oxidative stress in the pathogenesis of breast cancer, and regulatory effects of melatonin have recently been indicated in vivo and in vitro (13). In this study, we aimed to evaluate clinical effects of melatonin in breast cancer patients, focusing on oxidative stress and inflammation biomarkers, and pathological findings of the tumor.

\section{Methods}

\section{Trial design and participants}

The current study was a randomized double-blind placebo-controlled clinical trial, registered in the Iranian registry of clinical trials (http://www.irct.ir: IRCT20171105037262N5), performed at Shahid-Beheshti hospital affiliated to Kashan University of Medical Sciences (KAUMS), Kashan, Iran, among 40 subjects with breast cancer at any stage undergoing neoadjuvant chemotherapy aged $>25$ years old between January 2020 and June 2021. Exclusion criteria were as follows: having renal and liver failure abnormal blood test.

\section{Study design}

Firstly, all subjects randomly divided into two groups to receive either melatonin supplements $(n=20)$ or placebo $(n=20)$ for 24 weeks. Every night one hour prior to sleep, patients will orally receive two $5 \mathrm{mg}$ capsules of melatonin for 24 weeks. The appearance of the placebo was indistinguishable in color, shape, size, and packaging, smell and taste from the melatonin capsule. All capsules were produced by Zahravi Pharmaceutical Company (Tabriz, Iran). Subjects were requested not to change their ordinary physical activity and not to take any nutritional supplements during the 24-week trial. 


\section{Treatment Adherence}

To evaluate the compliance, the remaining supplements were counted and subtracted from the amount of supplements provided to the subjects. To increase compliance, all subjects received short messages on their cell phones every day to remind them about taking the capsules.

\section{Biochemical assessment}

Ten milliliters fasting blood samples were collected at baseline and after the 24-week intervention at Kashan reference laboratory in a fasting status. Then, the samples were stored at $-80^{\circ} \mathrm{C}$ before analysis. Serum C-reactive protein (CRP) values were assessed by commercial ELISA kit (LDN, Nordhorn, Germany) with inter- and intra-assay CVs of 4.5 to $6.4 \%$, respectively. The plasma NO using Griess method (14), TAC by the use of ferric reducing antioxidant power developed by Benzie and Strain (15), GSH using the method of Beutler et al. (16) and MDA concentrations by the thiobarbituric acid reactive substances spectrophotometric test (17) were determined. All inter- and intra-assay CVs for NO, TAC, GSH and MDA values were less than $4 \%$.

\section{Sample size}

Using a formula suggested for clinical trials, having 25 subjects in each group were adequate while considering a type one error (a) of 0.05 and type two error ( $\beta$ ) of 0.20 (power=80\%), $283.7 \mathrm{ng} / \mathrm{mL}$ as SD and $290.0 \mathrm{ng} / \mathrm{mL}$ as the mean distinction (d) of hsCRP as the key variable (18). Assuming 5 dropouts in each group, the final sample size was determined to be 20 subjects in each group.

\section{Randomization}

Randomization and allocation were conducted as blindness using computer-generated random numbers.

\section{Statistical methods}

To evaluate whether the study variables were normally distributed or not, we used the Kolmogrov-Smirnov test. To assess the effects of melatonin supplementation on biomarkers of inflammation and oxidative stress, we used one-way repeated measures analysis of variance. The P-value of $<0.05$ were considered statistically significant. All statistical analyses used the Statistical Package for Social Science version 18 (SPSS Inc., Chicago, Illinois, USA).

\section{Results}

In the current study, all 33 subjects with breast cancer [melatonin $(n=17)$ and placebo $(n=16)$ ] completed the trial (Fig. 1). On average, the rate of compliance in the present study was high, such that higher than $90 \%$ of capsules were taken throughout the study in both groups. No side effects were reported following consumption of melatonin supplements in subjects with breast cancer throughout the study.

After the 24-week intervention, compared with the placebo, melatonin supplementation significantly increased plasma TAC (+21.3 \pm 26.2 vs. $-6.3 \pm 34.5 \mathrm{mmol} / \mathrm{L}, \mathrm{P}=0.007)$, and decreased plasma MDA concentrations $(-0.2 \pm 0.3 \mathrm{vs} .+0.1 \pm 0.2 \mu \mathrm{mol} / \mathrm{L}, \mathrm{P}=0.002)$ (Table 1). We did not observe any significant effect of melatonin supplementation on other biomarkers of oxidative stress.

\section{Discussion}

In the present study, melatonin intake for 24 weeks in patients with breast cancer had beneficial effects on TAC and MDA levels, but did not affect other biomarkers of oxidative stress. In animal models, melatonin, especially in combination with metformin, suppressed DMBA-mediated mammary tumor growth through apoptosis stimulation in cancer cells (19). Pioglitazone and melatonin combination did not considerably change the basic tumor growth parameters; however, histopathological findings revealed a reduction in the high-grade tumors proportion in vivo (20). 
In order to examine the clinical potential of melatonin against breast cancer, some studies have been conducted. In 1995, Lissoni et al. evaluated the efficacy of concomitant melatonin $(20 \mathrm{mg} / \mathrm{d})$ treatment in metastatic breast cancer patients progressed in response to tamoxifen alone. The treatment was well tolerated and lead to a partial response in $28.5 \%$ of the subjects. In addition, no melatonin-mediated enhancement of tamoxifen toxicity was observed (21). Lissoni and colleagues conducted another study in 14 metastatic breast cancer patients treated by epirubicin $(25 \mathrm{mg} / \mathrm{m} 2$ i.v. at weekly intervals) and oral intake of melatonin (20 $\mathrm{mg} / \mathrm{d}$; starting 7 days before chemotherapy). The induction phase with melatonin mediated a platelet number normalization in 9 of 12 evaluable patients, and no further platelet decrease happened in chemotherapy. Of note, objective tumor regression was achieved in 5 of 12 cases, suggesting melatonin effectiveness in the cancer-associated thrombocytopenia treatment and to prevent chemotherapy-mediated platelet decline (22).

In another clinical study on breast cancer patients, melatonin (3 mg; daily) was shown well tolerated in a four-month course without any toxicity, but it did not affect circulating estradiol, IGFBP-3, or IGF-1 levels in postmenopausal females with breast cancer (23). In 2020, neuroprotective effect of melatonin on the chemotherapy-induced adverse events in breast cancer patients was evaluated. In this study, melatonin ameliorated executive function on trial making test (TMT) scores, increased episodic memory (delayed and immediate) and recognition on Rey Auditory-Verbal Learning Test (RAVLT), and enhanced verbal fluency in the orthographic Controlled Oral Word Association Test (COWAT). At the end of treatment, changes in brain-derived neurotrophic factor (BDNF) and TrkB were inversely correlated with sleep quality and depressive symptoms, but not with the TMT scores (24).

\section{Conclusions}

Today, the treatment of human cancers, especially breast cancer, is a global concern and much attention has been attracted to this issue. Melatonin, a neurohormone with multiple properties in various pathologies, has been well-established as an anticancer agent in many preclinical investigations; however, there is little data on its efficacy in the treatment of breast cancer patients in clinical practice. Therefore, we aimed to design the present trial to investigate melatonin effects on biomarkers of inflammation and oxidative stress. We showed that melatonin intake for 24 weeks in patients with breast cancer had beneficial effects on TAC and MDA levels, but did not affect other biomarkers of oxidative stress. Overall, more clinical and preclinical trials are needed for better evaluation on melatonin effects based on underlying mechanisms

\section{Declarations}

\section{Funding}

No funding was received for this study.

\section{Authors' contributions}

AHM contributed in conception, design, statistical analysis and drafting of the manuscript. FH, NM, RMA and MHP contributed in data collection and manuscript drafting. All authors approved the final version for submission.

\section{Availability of data and materials}

The datasets utilized and analyzed during the current investigation are available from the corresponding author upon any reasonable request.

\section{Ethics approval and consent to participate}

This research was done in accordance with the Declaration of Helsinki and informed consent was taken from all subjects. The research was approved by the ethics committee of KAUMS.

\section{Consent for publication}

Not applicable. 


\section{Competing interests}

The authors declare that they have no competing interests.

\section{Acknowledgments}

Not applicable.

\section{Clinical trial registration number}

http://www.irct.ir:

\section{References}

1. García-Aranda M, Redondo M. Protein Kinase Targets in Breast Cancer. International journal of molecular sciences. 2017;18(12).

2. Sun YS, Zhao Z, Yang ZN, Xu F, Lu HJ, Zhu ZY, et al. Risk Factors and Preventions of Breast Cancer. Int J Biol Sci. 2017;13(11):1387-97.

3. Ye JC, Formenti SC. Integration of radiation and immunotherapy in breast cancer - Treatment implications. Breast (Edinburgh, Scotland). 2018;38:66-74.

4. Cipolla-Neto J, Amaral FGD. Melatonin as a Hormone: New Physiological and Clinical Insights. Endocrine reviews. 2018;39(6):990-1028.

5. Seely D, Wu P, Fritz H, Kennedy DA, Tsui T, Seely AJ, et al. Melatonin as adjuvant cancer care with and without chemotherapy: a systematic review and meta-analysis of randomized trials. Integrative cancer therapies. 2012;11(4):293-303.

6. Mahmood D. Pleiotropic Effects of Melatonin. Drug research. 2019;69(2):65-74.

7. Hanikoglu A, Kucuksayan E, Akduman RC, Ozben T. A Review on Melatonin's Effects in Cancer: Potential Mechanisms. Anticancer agents in medicinal chemistry. 2018;18(7):985-92.

8. Mazzoccoli G, Carughi S, De Cata A, La Viola M, Vendemiale G. Melatonin and cortisol serum levels in lung cancer patients at different stages of disease. Medical science monitor : international medical journal of experimental and clinical research. 2005;11(6):Cr284-8.

9. Karasek M, Kowalski AJ, Suzin J, Zylinska K, Swietoslawski J. Serum melatonin circadian profiles in women suffering from cervical cancer. Journal of pineal research. 2005;39(1):73-6.

10. de Castro TB, Bordin-Junior NA, de Almeida EA, de Campos Zuccari DAP. Evaluation of melatonin and AFMK levels in women with breast cancer. Endocrine. 2018;62(1):242-9.

11. Scholtens RM, van Munster BC, van Kempen MF, de Rooij SE. Physiological melatonin levels in healthy older people: A systematic review. Journal of psychosomatic research. 2016;86:20-7.

12. Magri F, Sarra S, Cinchetti W, Guazzoni V, Fioravanti M, Cravello L, et al. Qualitative and quantitative changes of melatonin levels in physiological and pathological aging and in centenarians. Journal of pineal research. 2004;36(4):256-61.

13. Amin N, Shafabakhsh R, Reiter RJ, Asemi Z. Melatonin is an appropriate candidate for breast cancer treatment: Based on known molecular mechanisms. J Cell Biochem. 2019;120(8):12208-15.

14. Tatsch E, Bochi GV, Pereira Rda S, Kober H, Agertt VA, de Campos MM, et al. A simple and inexpensive automated technique for measurement of serum nitrite/nitrate. Clin Biochem. 2011;44(4):348-50.

15. Benzie IF, Strain JJ. The ferric reducing ability of plasma (FRAP) as a measure of "antioxidant power": the FRAP assay. Anal Biochem. 1996;239(1):70-6.

16. Beutler E, Gelbart T. Plasma glutathione in health and in patients with malignant disease. J Lab Clin Med. 1985;105(5):581-4.

17. Janero DR. Malondialdehyde and thiobarbituric acid-reactivity as diagnostic indices of lipid peroxidation and peroxidative tissue injury. Free Radic Biol Med. 1990;9(6):515-40. 
18. Asemi Z, Khorrami-Rad A, Alizadeh SA, Shakeri H, Esmaillzadeh A. Effects of synbiotic food consumption on metabolic status of diabetic patients: a double-blind randomized cross-over controlled clinical trial. Clin Nutr. 2014;33(2):198-203.

19. Bojková B, Kajo K, Kisková T, Kubatka P, Žúbor P, Solár P, et al. Metformin and melatonin inhibit DMBA-induced mammary tumorigenesis in rats fed a high-fat diet. Anti-cancer drugs. 2018;29(2):128-35.

20. Bojková B, Orendáš $P$, Kajo K, Kubatka P, Výbohová D, Bálentová S, et al. Role of high-fat diet on the effect of pioglitazone and melatonin in a rat model of breast cancer. European journal of cancer prevention : the official journal of the European Cancer Prevention Organisation (ECP). 2016;25(5):395-403.

21. Lissoni P, Barni S, Meregalli S, Fossati V, Cazzaniga M, Esposti D, et al. Modulation of cancer endocrine therapy by melatonin: a phase II study of tamoxifen plus melatonin in metastatic breast cancer patients progressing under tamoxifen alone. British journal of cancer. 1995;71(4):854-6.

22. Lissoni P, Tancini G, Paolorossi F, Mandalà M, Ardizzoia A, Malugani F, et al. Chemoneuroendocrine therapy of metastatic breast cancer with persistent thrombocytopenia with weekly low-dose epirubicin plus melatonin: a phase II study. Journal of pineal research. 1999;26(3):169-73.

23. Schernhammer ES, Giobbie-Hurder A, Gantman K, Savoie J, Scheib R, Parker LM, et al. A randomized controlled trial of oral melatonin supplementation and breast cancer biomarkers. Cancer causes \& control : CCC. 2012;23(4):609-16.

24. Palmer ACS, Zortea M, Souza A, Santos V, Biazús JV, Torres ILS, et al. Clinical impact of melatonin on breast cancer patients undergoing chemotherapy; effects on cognition, sleep and depressive symptoms: A randomized, double-blind, placebocontrolled trial. PloS one. 2020;15(4):e0231379.

\section{Tables}

Table 1. Biomarkers of inflammation and oxidative stress at baseline and after the 24-week intervention in subjects with breast cancer

\begin{tabular}{|llllllll|}
\hline \multicolumn{7}{|c}{ Placebo group $(\mathrm{n}=20)$} & \multicolumn{5}{l}{ Melatonin group $(\mathrm{n}=20)$} \\
& Baseline & End-of-trial & Change & Baseline & End-of-trial & Change & $\mathrm{P}^{1}$ \\
\hline $\mathrm{CRP}(\mathrm{mg} / \mathrm{L})$ & $4.7 \pm 1.1$ & $5.0 \pm 1.2$ & $0.3 \pm 0.4$ & $5.1 \pm 1.0$ & $5.2 \pm 0.9$ & $0.1 \pm 0.4$ & 0.10 \\
\hline $\mathrm{NO}(\mu \mathrm{mol} / \mathrm{L})$ & $64.9 \pm 4.3$ & $65.0 \pm 4.1$ & $0.1 \pm 2.7$ & $65.3 \pm 7.0$ & $65.8 \pm 8.0$ & $0.5 \pm 5.1$ & 0.78 \\
\hline $\mathrm{TAC}(\mathrm{mmol} / \mathrm{L})$ & $659.8 \pm 158.0$ & $653.5 \pm 155.7$ & $-6.3 \pm 34.5$ & $694.8 \pm 131.8$ & $716.1 \pm 136.3$ & $21.3 \pm 26.2$ & 0.007 \\
\hline GSH $(\mu \mathrm{mol} / \mathrm{L})$ & $582.6 \pm 119.9$ & $577.9 \pm 125.4$ & $-4.7 \pm 31.9$ & $605.3 \pm 154.8$ & $599.0 \pm 151.4$ & $-6.3 \pm 21.0$ & 0.85 \\
\hline MDA $(\mu \mathrm{mol} / \mathrm{L})$ & $3.9 \pm 1.0$ & $4.0 \pm 1.1$ & $0.1 \pm 0.2$ & $3.6 \pm 0.9$ & $3.3 \pm 0.7$ & $-0.2 \pm 0.3$ & 0.002 \\
\hline
\end{tabular}

All values are means \pm SDs.

${ }^{1} \mathrm{P}$ values represent the time $\times$ group interaction (computed by analysis of the one-way repeated measures ANOVA).

GSH, total glutathione; hs-CRP, C-reactive protein; MDA, malondialdehyde; NO, nitric oxide; TAC, total antioxidant capacity.

\section{Figures}




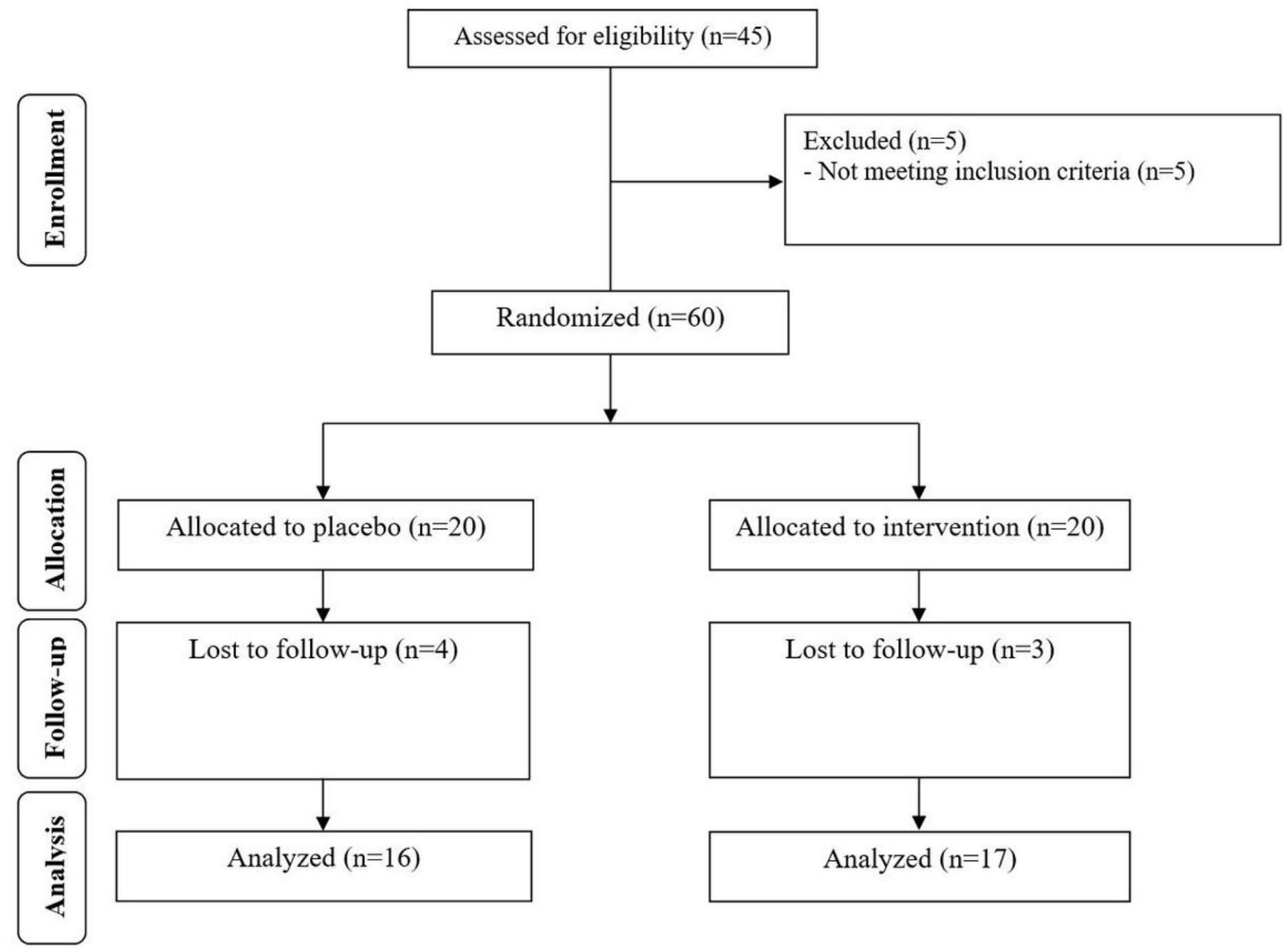

Figure 1

Summary of patient flow diagram. 